\title{
Out of hours workload management: Bayesian inference for decision support in secondary care
}

\author{
Iker Perez ${ }^{\mathrm{a}, *}$, Michael Brown ${ }^{\mathrm{a}}$, James Pinchin ${ }^{\mathrm{a}}$, Sarah Martindale ${ }^{\mathrm{a}}$, Sarah \\ Sharples $^{\mathrm{b}}$, Dominick Shaw ${ }^{\mathrm{c}}$, John Blakey $^{\mathrm{d}}$ \\ ${ }^{a}$ Horizon Digital Economy Research Institute, The University of Nottingham, Nottingham \\ Geospatial Building, Jubilee Campus, Wollaton Road, Nottingham NG8 1BB, UK. \\ ${ }^{b}$ Faculty of Engineering, The University of Nottingham, Room B25 Coates Building, \\ University Park, Nottingham NG7 2RD, UK \\ ${ }^{c}$ Faculty of Medicine 83 Health Sciences, The University of Nottingham, Room B21 \\ Nottingham City Hospital, Hucknall Road, Nottingham NG5 1PB UK \\ ${ }^{d}$ Department of Clinical Sciences, Liverpool School of Tropical Medicine, Pembroke Place, \\ Liverpool L3 5QA, UK
}

\begin{abstract}
Objective: In this paper, we aim to evaluate the use of electronic technologies in Out of Hours $(\mathrm{OoH})$ task-management for assisting the design of effective support systems in health care; targeting local facilities, wards or specific working groups. In addition, we seek to draw and validate conclusions with relevance to a frequently revised service, subject to increasing pressures.

Methods and Material: We have analysed 4 years of digitised demand-data extracted from a recently deployed electronic task-management system, within the Hospital at Night setting in two jointly coordinated hospitals in the United Kingdom. The methodology employed relies on Bayesian inference methods and parameter-driven state-space models for multivariate series of count data.

Results: Main results support claims relating to (i) the importance of datadriven staffing alternatives and (ii) demand forecasts serving as a basis to intelligent scheduling within working groups. We have displayed a split in workload patterns across groups of medical and surgical specialities, and sustained assertions regarding staff behaviour and work-need changes according to shifts or days of the week. Also, we have provided evidence regarding the relevance of day-to-day planning and prioritisation.

Conclusions: The work exhibits potential contributions of electronic tasking alternatives for the purpose of data-driven support systems design; for scheduling, prioritisation and management of care delivery. Electronic tasking technolo-
\end{abstract}

\footnotetext{
*Corresponding author. Iker Perez, Nottingham Geospatial Building, Jubilee Campus, Wollaton Road, Nottingham NG8 1BB, UK. +44 (0) 7547284617.

Email addresses: iker.perez@nottingham.ac.uk (Iker Perez),

michael.brown@nottingham.ac.uk (Michael Brown), james.pinchin@nottingham.ac.uk

(James Pinchin), sarah.martindale@nottingham.ac.uk (Sarah Martindale),

sarah.sharples@nottingham.ac.uk (Sarah Sharples), dominic.shaw@nottingham.ac.uk

(Dominick Shaw), john.blakey@lstmed.ac.uk (John Blakey)
}

Preprint submitted to Artificial Intelligence in Medicine

August 2, 2016 
gies provide means to design intelligent systems specific to a ward, speciality or task-type; hence, the paper emphasizes the importance of replacing traditional pager-based approaches to management for modern alternatives.

Keywords: Healthcare Management, Multivariate Time Series, Count Data, Out of Hours, Graphical Model 


\section{Response to reviewers}

General Comments: We are grateful to all reviewers for taking the time to review our paper and make a number of helpful comments that we believe have significantly improved the quality of the manuscript. All comments have been, 5 according to their nature, merged, described and addressed here:

Comment 1: The major concern shared by reviewers relates to a lack practical relevance of the results presented; for instance, managerial conclusions are not well exploited, the paper lacks a managerial perspective of the output from professionals involved in the study, or results do not extrapolate to different settings neither is it clear why they support the decision making.

Response to Comment 1: The authors agree that the previous version of the manuscript offered a mostly descriptive study of the data and lacked relevant interpretations and conclusions drawn from results. In addition, we believe we were not successful in clearly conveying the aims and scope of our work; yet, we have made the efforts to undertake the alterations necessary in order to correct these problems.

Nationwide hospital settings are indeed subject to disparate workload pressures; these may drastically vary within distant geographical regions and in relation to specialities covered by each facility. The focus of our research team

20 and this work relates to support systems for effective management that can target local facilities and working groups. This is indeed a great problem, in that policy alterations and intelligent design of support systems (for instance, for rota design) are generally based on nationwide statistics of global admission and consultation counts, which lack the ability to represent the working loads of

25 small wards or facilities. Hence, it is important to provide evidence regarding the additive value of switching from old pager-based management systems to more modern electronic alternatives; being the most relevant one the ability to gain a detailed understanding of workload pressures for further design of data-driven and facility-oriented prioritisation, scheduling and management methods. We

so have thus made alterations (primarily in introduction and abstract) and added relevant references to emphasize these important matters.

Also, such a long data acquisition can certainly allow for certain generalisation of conclusions. We collaborate with nationwide institutions (Royal College of Physicians) and medical consultants who supervised the data acquisition over

35 these years. In this new version of the manuscript, we have scrutinized professionals and put a big emphasis on the extent up to which results generalise to different facilities. We have significantly extended discussions of results and we have drawn relevant conclusions not restricted to the study (specially in results and discussion sections).

Comment 2: While technically well explained, there is a lack of comprehensibility in the modelling approach. A worked example would be welcome, along with justifications of design decisions taken. Also, the parameter optimization routine is not explained. 
Response to Comment 2: We thank the reviewer for pointing out the need 45 for better comprehensibility within the model set-up. Following the suggestion, a worked example for the global series of task-demand counts has been included; hence, Section 2 has been altered in order to justify and provide practical examples for design decisions taken. In addition, we have included a summary set of steps for the simulation-based parameter-optimization routine employed; thus

50 describing marginal posterior distributions for all parameters within the model (end of Section 2).

Comment 3: Taking a single modelling approach is problematic. I suggest an extension of the work with related work section, where other approaches in terms of time series modelling are reviewed and one or two most appropriate are compared on the same data.

Response to Comment 3: In this new version of the manuscript, we have included a section with references to previous work in modelling series of count data within a medical domain. While literature on time series modelling is extensive and there exist numerous approaches to the matter, in terms of count ${ }_{60}$ data series this is usually reduced to observation and parameter driven settings employing Poisson errors; and hence fall within the family of state space models (Gaussian approximations are also common). Thus, we have reflected our thoughts on this matter and resorted to two common alternative methods in order to test congruency on results within a predictive setting (see also Comment $4)$.

Comment 4: The data analysis should be extended including a predictive model and correlation analysis among different variables.

Response to Comment 4: Indeed, testing the predictability of future demand is of special relevance as a basis to justify the usefulness and thus importance 70 for the deployment of such data collection methodology within local healthcare facilities. Also, it can contribute to growing debates discussing the need for data-driven methods for intelligent rota-design. We thank the reviewer for identifying this and we have now included a whole subsection focused on predictability; this includes predictive comparisons over different time-horizons, 75 employing forward-filtering approaches for latent dynamics in the model. Additionally, we have established comparisons with observation-driven methodology and standard approaches based in generalized linear models (see also Comment 3). Also, a correlation analysis within model parameters was not included, since all parameter chains within the MCMC routine were fully uncorrelated; yet, we so have made a explicit mention to this fact in Section 5

Comment 5: The paper is generally well written and informative. It is statistical in nature, so I am not sure that it falls under a journal devoted to artificial intelligence in medicine. Could the results be used in some type of machine learning or other approach to intelligent scheduling? this would make 85 it more relevant to the journal.

The paper offers a rather statistical analysis of a data set with aims of dis- 
cussing the additive value regarding embedded technologies for management support. We note that forecasting of work-demand is the basis to any intelligent method aiming to provide support for scheduling, prioritisation or management of care (see also Comment 4). In this regard, the manuscript provides strong evidence concerning the power of such tasking information within local facilities for (i) estimation of future demand (ii) enabling the understanding of workload within speciality specific wards or working groups. Thus, the authors understand that the target audience for this manuscript is the community of 95 researchers with a focus on decision support and expert systems for healthcare; rather than a statistical community with greater interest on methodology and the study of causality relationships in data. We thank the reviewer for bringing this to our attention, and we have now made the efforts to emphasize our perspective on the matter within the manuscript.

Final Comments: Finally, we have altered the manuscript in order to include full postal addresses; adapt the abstract to journal structure; remove capitalisation within words in titles, subtitles and figures; compress reference citations within text; and complete references within reference list. 


\section{Introduction}

105 ([1, 2]). Patient admissions are rising ([3]), and the number of available beds is falling (4]); simultaneously, the complexity of conditions and treatments is increasing ([5]). Hence, healthcare systems must undergo major changes and optimise the use of limited resources. For $75 \%$ of the working week hospitals are staffed by a skeletal team (6]), and care must be delivered by a small and often junior group of clinicians over a wide range of medical specialities; frequently in large and complex sites ([7]). Decreasing budgets ([8]), tighter controls of working hours $([9])$ and the desire 115 for separation of work and private life ([10, 11]) have lead to a shrinkage of $\mathrm{OoH}$ working teams. Hence, in order to deliver safe healthcare of a consistently high quality the provision of this service is frequently revised ([12 14]), often without underlying comprehensive data or understanding of the demand placed on clinical teams.

Extensive research has been concerned with the study of expert and knowledgebased systems in healthcare management. This includes logistics, resource scheduling or estimation of service demands, and we refer the reader to [15 17] (and references therein) for some examples of this work. In particular, quantitative demand-forecasting studies focused on patient volumes (e.g. [18]) have conroles in understanding demand loads. Additionally, the study of explanatory covariates in admission volumes has proved helpful in order to identify social pressures on workload (e.g. [19]). However, research restricted to global admission and consultation counts is insufficient in order to inform policy on local

fff management; note that different patients receiving unrelated treatment over several medical disciplines put disparate workload pressures on specialist staff groups and grades; moreover, such pressures may vary drastically within distant geographical regions.

Hence, there is a need to employ modern embedded technologies for the design of effective management support systems that can target local facilities and specific working groups ([20]); examples of such work include [21] and [22]. In this paper, we explore the use of electronic task-management for the study of $\mathrm{OoH}$ workload in secondary facilities. The purpose is twofold; on one hand, to exhibit the additional value that such tasking data provides in combination with ${ }_{140}$ modern machine learning methodology, for supporting intelligent scheduling, prioritisation and management of care delivery. On the other hand, to draw and validate conclusions with managerial relevance not restricted to the timewindow and medical facilities covered in this study. We note that task-demand and completion numbers offer a better representation of workload, as opposed to admission or consultation counts; a lack of available sources has precluded previous quantitative studies of this kind.

To address these matters, we employ a state-space graphical model allowing for the extraction of patterns of cyclic variation in task-demand. This provides 
a useful framework to treat long series of observed multivariate counts, assuming independent observations conditionally on the values of a latent process. It allows to not underestimate true serial dependencies and control for discreteness and over-dispersion (23); moreover, link functions may allow for intuitive interpretations of covariates' effects (e.g. [24]). Thus, by means of a latent parameter-driven model, we show it is possible to draw inference on contem155 porary and serial correlations on demand, over different medical and surgical specialities within a local facility. We also exhibit the ability to quantify future demand pressures, and we compare results with approaches relying on common methodology. Finally, we offer a summary of relevant conclusions as scrutinized by local medical staff, consultants and nation-wide healthcare organizations.

The data collected for this purpose was gathered from two major university hospitals, which combined provide secondary healthcare to over 2.5 million residents in the United Kingdom. In both hospitals involved, tasks for the team are requested, assigned and managed via web and mobile device interfaces; and the data is collected at each stage of this process, allowing work-demand to be monitored and analysed. The dataset used for this work contained 652,585 task requests and covers the period from January 2012 to December 2015.

Main results in the paper identify shared characteristics of OoH workload and display a significant split between medical and surgical specialities. Also, strong serial dependencies in demand series and a fast-decreasing predictive power over increasing time-windows emphasize the importance of short-term scheduling and prioritisation. Moreover, results support claims relating to both the importance of data-driven local staffing and work-demand forecasts serving as a basis to intelligent scheduling support. Patient and administrative needs vary significantly according to the day of the week or shift; notably, weekend planning must account for the variation between medical and surgical wards and bank holidays need to be treated as weekends; yet, workforce should (subject to few exceptions) be similar all year-round.

The rest of the paper is organised as follows; in Section 2 we review literature on state-space models with applications in time-series analysis, and explain 180 the model employed in this study. Section 3 offers a discussion on alternative approaches and related work within a medical context. A description and summary of the data is offered in Section 4 . In Section 5 we report results in the paper, and finally we offer conclusions and discuss policy recommendations in Section 6

\section{State-space models for multivariate count data}

State-space models are a class of probabilistic graphical models (see [25]) that have found applications in time-series analysis for supply chain planning [26], text and music analysis ([27]), econometrics ([28]) or political science ([29]), to name a few. In particular, they allow us to describe the dependence between 190 continuous latent state variables and discrete observed counts in terms of some probabilistic distribution; hence, they pose a useful mean to relate electronic tasking information with patterns of workload in healthcare facilities. 
These models are either observation or parameter driven, and can extend generalized linear models by incorporating latent autoregressive processes within the conditional mean function (cf. [24, 30]); thus introducing both auto-regression and over-dispersion. Less common multivariate extensions can handle both contemporary and serial correlations, and therefore tackle questions not addressed by marginal models (cf. [31, 32]). In general, these models employ dynamic factors or vector auto-regressions at the latent level, and various technical examples of this work can be found in $([28,31,33])$ and references therein.

Let $\boldsymbol{y}_{t} \in \mathbb{N}_{0}^{n}$ denote a vector of task counts as observed at day $t$; in our application, this reflects counts over $n$ different medical and surgical specialities within the two hospitals. We now formulate a model such that $\boldsymbol{y}_{t}$ is drawn from a family of conditionally-independent Poisson distributions, such that

$$
y_{t, i} \mid h_{t}, \boldsymbol{\mu}_{t} \sim \mathcal{P}\left(h_{t} \cdot \mu_{t, i}\right), \quad \text { for } i \in\{1, \cdots, n\},
$$

and

$$
\log \left(\boldsymbol{\mu}_{t}\right)=\sum_{j} \boldsymbol{f}_{j}(t)+\boldsymbol{\nu}_{t}, \quad \text { for } t \in\{1, \cdots, T\} .
$$

Here, $\boldsymbol{\mu}_{t} \in \mathbb{R}_{+}^{n}$ denotes a latent rate vector of hourly-tasks, and $h_{t} \in\{16,24\}$ indicates the working hours of the day. Thus, we aim to capture the relation within daily workload and temporal or autoregressive patterns by means of a log-link function. In the following, we discuss the components of the model along with a simplified example for the univariate series of global counts in Figure 1 .

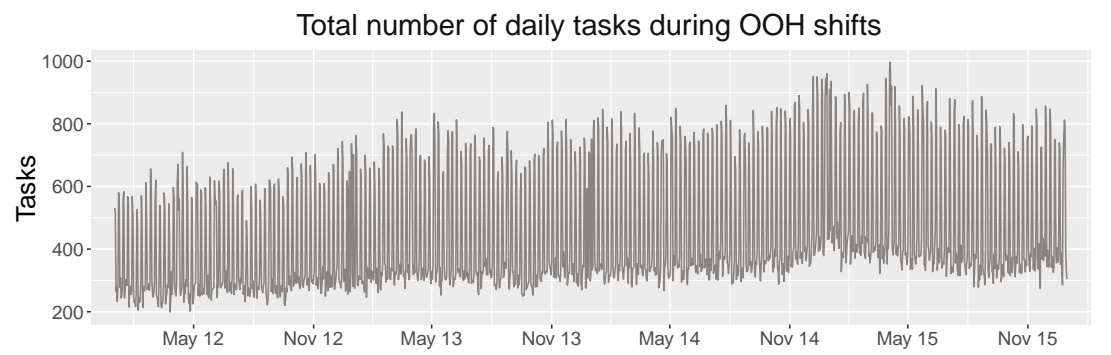

Figure 1: Joint time series of task-demand across both hospitals and specialities covered in the study.

\subsection{Temporal trends in task-demand}

The family $\left\{\boldsymbol{f}_{j}(t) \in \mathbb{R}^{n}: j \geq 1\right\}_{t \geq 0}$ are vector sequences defined to capture cyclic trends of task-demand; we note that these are log-multiplicative factors scaling hourly-task rates and hence the expected demand in (1). Particularly, $\boldsymbol{f}_{1}(t)$ includes $n \times 2$ coefficients $\boldsymbol{\beta}_{1}$ that represent independent linear trends of workload over different specialities; for instance, in Figure 2 (Left) we observe a credible interval for a linear growing trend in global task-demand within this 
data set, there we notice an approximate $48 \%$ increase on demand over a 4 year period. Next,

$$
\boldsymbol{f}_{j}(t)=\sum_{k=1}^{K_{j}} \boldsymbol{\beta}_{j, k} \cdot\left(\cos \left(\frac{2 \pi k t}{P_{j}}\right), \sin \left(\frac{2 \pi k t}{P_{j}}\right)\right)^{\prime}, \quad j \in\{2,3\}
$$

are Fourier terms with periodicities $P_{2}=7$ and $P_{3}=365$. These are frequently employed (c.f. [28, 33]) in both frequentist and Bayesian settings in order to capture weekly and year-round patterns. It is noticeable in Figure 2 (center) 210 that global $\mathrm{OoH}$ demand is highly influenced by the divide within weekday and weekend shifts (mostly explained due to further daylight hours during weekends); additionally, a mild yet significant decay is observed within the months of August and September (plot on the right).
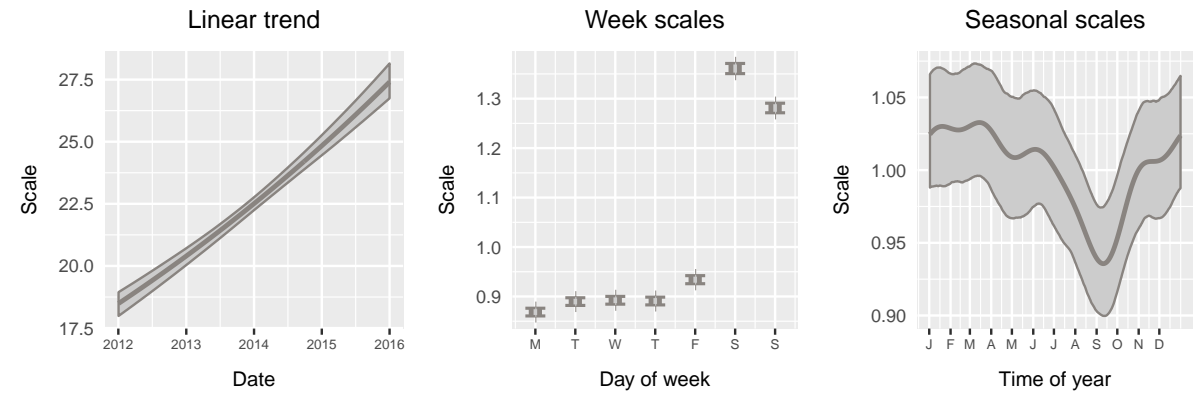

Figure 2: Graphical summary of exponentiated scales on global hourly-demand, across both hospitals and specialities covered in the study. Credible intervals shown at a $95 \%$ level.

Also, $\boldsymbol{f}_{4}(t)$ includes $n$ coefficients $\boldsymbol{\beta}_{4}$ allowing to further scale demand during Bank Holidays. In our worked example, this is a single parameter and its exponential has mean value 1.44 . We finally note that

$$
\boldsymbol{\beta}=\left\{\boldsymbol{\beta}_{j} ; j \in\{1, \cdots, 4\}\right\}
$$

accounts for $n \times\left(3+2\left(K_{2}+K_{3}\right)\right)$ regression parameters.

\subsection{Autoregressive trends in task-demand}

In addition to seasonal trends, $\left\{\boldsymbol{\nu}_{t} \in \mathbb{R}^{n}: t \geq 1\right\}$ defines a sequence of latent effects accommodating possible contemporaneous and serial dependencies in counts, within a parameter driven framework. This is such that

$$
\boldsymbol{\nu}_{t} \sim \mathcal{N}\left(\operatorname{diag}(\boldsymbol{\delta}) \cdot \boldsymbol{\nu}_{t-1}, \Sigma\right), \quad \text { for } t \geq 2,
$$

and $\boldsymbol{\nu}_{1} \sim \mathcal{N}(\mathbf{0}, \Sigma)$. Thus, it defines a vector auto-regression with a basic serial dependence structure, controlling for likely epidemic departures in marginal workload trends. Here, $\Sigma$ denotes a positive-definite covariance matrix incorporating correlation and over-dispersion within the model. Furthermore, in order 
to guarantee stationarity we impose $\left|\delta_{i}\right|<1$ for all $i \in\{1, \cdots, n\}$, and note that matrix $\Theta$ with

$$
\Theta_{i, j}=\Sigma_{i, j} /\left(1-\delta_{i} \delta_{j}\right) \quad \text { for } i, j \in\{1, \cdots, n\} .
$$

denotes the stationary covariance matrix in (3). In Figure 3, we observe the sequence of latent effects for global demand during 2012; note that in the univariate case, the above formulation reduces to an $\mathrm{AR}(1)$ model with no constant, single parameter and white noise. In the figure, we notice positive autoregressive dynamics; its factor has mean 0.79 with a $95 \%$ credible interval of $(0.74,0.83)$.

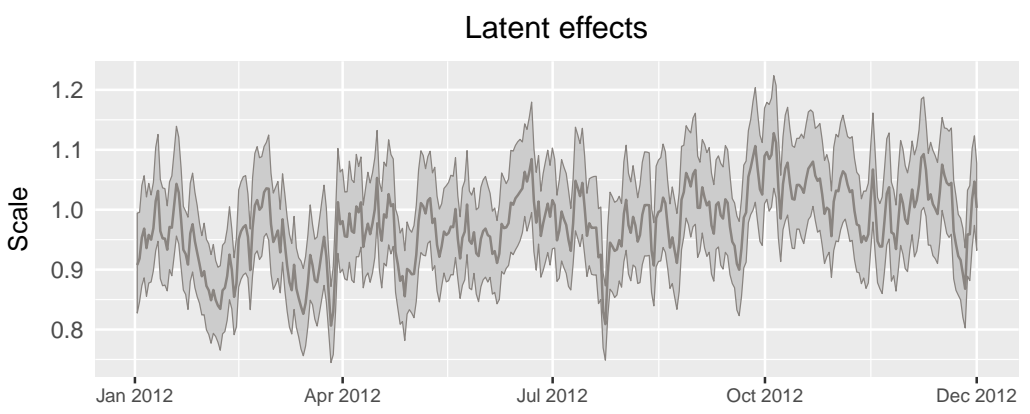

Figure 3: Series of latent effects in global hourly task-demand during 2012.

\subsection{Graphical model, moments and parameter estimation}

A graphical representation of the model in template notation is offered in Figure 4 in the case where $\boldsymbol{\delta}=\mathbf{0}$, the above formulation reduces to a variation of the model for correlated count data presented in [34. Under this setting, it can be readily verified that

$$
\hat{\boldsymbol{y}}_{t}:=\mathbb{E}\left[\boldsymbol{y}_{t} \mid h_{t}, \boldsymbol{f}, \boldsymbol{\delta}, \Sigma\right]=h_{t} \cdot \exp \left(\frac{1}{2} \cdot \tilde{\Theta}+\sum_{j=1}^{4} \boldsymbol{f}_{j}(t)\right),
$$

and

$$
\operatorname{Cov}\left(\boldsymbol{y}_{t} \mid h_{t}, \boldsymbol{f}, \boldsymbol{\delta}, \Sigma\right)=\left(\hat{\boldsymbol{y}}_{t} \cdot \hat{\boldsymbol{y}}_{t}^{\prime}\right) \circ\left(e^{\Theta}-1\right)+\operatorname{diag}\left(\hat{\boldsymbol{y}}_{t}\right),
$$

for all $t \in\{1, \cdots, T\}$, where $\tilde{\Theta}$ stands for the entries in the main diagonal of $\Theta$ and $\circ$ denotes the Hadamard product. Thus, main moments can be approximated according to laws of iterated expectation, with samples from the full posterior distribution of autoregressive parameters, the covariance structure, and sequences

$$
\left\{e^{f_{j}(t)}: j \in\{1, \cdots, 4\}, t \in\{1, \cdots, T\}\right\}
$$




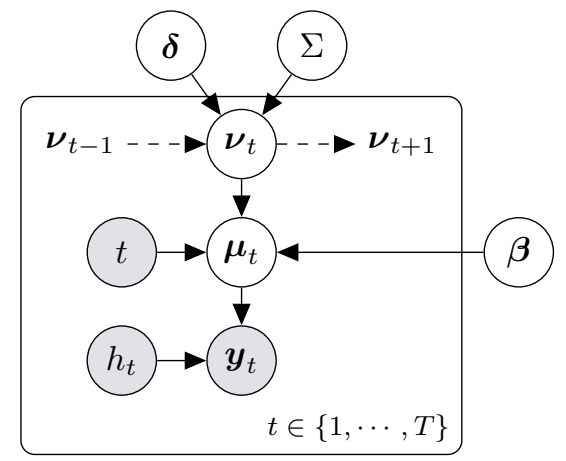

Figure 4: Graphical representation of the relation within counts and the latent structure in the model.

which we recall are multiplicative scaling factors for $\boldsymbol{\mu}$ in (1).

In this work, the focus is given to (i) the study of distributional properties of model components, in order to unveil workload patterns during OoH shifts, and (ii) the assessment of the ability to quantify future task-demand, for providing support within intelligent management design. We note that analytical inference is intractable due to non-conjugacy between the exponential family and the normal latent process; thus, we resort to stochastic simulation and learning on latent states and parameters $\{\boldsymbol{\beta}, \boldsymbol{\delta}, \Sigma\}$ is carried out through Markov Chain Monte Carlo, employing Metropolis-Hastings (M-H) updates within blocked Gibbs steps. Starting values can be extracted ignoring the latent dynamic structure, and using standard optimization routines for classic likelihood-based estimation methods. Also, parameter priors for $\boldsymbol{\beta}$ and $\boldsymbol{\delta}$ in (2)-(3) are assumed uninformative, and a Wishart prior distribution is used for the precision matrix $\Sigma^{-1}$ in (3). In summary,

a) Latent vector dynamics in $\boldsymbol{\nu}_{t}$ are updated by M-H, with target density

$$
\begin{aligned}
\pi\left(\boldsymbol{\nu}_{t} \mid \boldsymbol{\nu}_{\backslash t}, \boldsymbol{f}, \boldsymbol{\delta}, \Sigma\right) \propto & \prod_{i=1}^{n} \exp \left(y_{t, i} \nu_{t, i}-h_{t} \exp \left(\sum_{j} f_{j, i}(t)+\nu_{t, i}\right)\right) \\
& \cdot \exp \left(-\frac{1}{2}\left(\boldsymbol{\nu}_{t}-\boldsymbol{\mu}\right)^{\prime} \Omega^{-1}\left(\boldsymbol{\nu}_{t}-\boldsymbol{\mu}\right)\right),
\end{aligned}
$$

for all $t \in\{2, \cdots, T-1\}$; cases $t \in\{1, T\}$ are straightforward. Here,

$$
\Omega=\left[\Sigma^{-1}+d(\boldsymbol{\delta}) \Sigma^{-1} d(\boldsymbol{\delta})\right]^{-1}, \quad \boldsymbol{\mu}=\Omega\left[\Sigma^{-1} d(\boldsymbol{\delta}) \boldsymbol{\nu}_{t-1}+d(\boldsymbol{\delta}) \Sigma^{-1} \boldsymbol{\nu}_{t+1}\right] .
$$

b) Parameters in $\boldsymbol{\beta}$ can be updated by M-H, with $n$ independent blocks of medical speciality-specific regressors, each with target density

$$
\pi\left(\boldsymbol{\beta}_{\cdot, i} \mid \boldsymbol{\nu}\right) \propto \prod_{t=1}^{T} \exp \left(y_{t, i} \sum_{j} f_{j, i}(t)-h_{t} \exp \left(\sum_{j} f_{j, i}(t)+\nu_{t, i}\right)\right),
$$


for all $i \in\{1, \cdots, n\}$

c) The precision matrix $\Sigma^{-1}$ is sampled from a Wishart distribution, such that

$$
\Sigma^{-1} \mid \boldsymbol{\nu}, \boldsymbol{\delta} \sim \mathcal{W}_{n}(m, V),
$$

with $m=m_{0}+T$ degrees of freedom, and scale matrix

$$
V=\left(V_{0}^{-1}+\boldsymbol{\nu}_{1} \boldsymbol{\nu}_{1}^{\prime}+\sum_{t=1}^{T-1}\left(\boldsymbol{\nu}_{t+1}-d(\boldsymbol{\delta}) \cdot \boldsymbol{\nu}_{t}\right)\left(\boldsymbol{\nu}_{t+1}-d(\boldsymbol{\delta}) \cdot \boldsymbol{\nu}_{t}\right)^{\prime}\right)^{-1}
$$

Here, $m_{0}$ and $V_{0}$ are the prior degrees of freedom and scale matrix for $\Sigma^{-1}$, respectively.

d) Finally, the vector of marginal autoregressive terms $\boldsymbol{\delta}$ is truncated normal, and may be updated so that

$$
\boldsymbol{\delta} \mid \boldsymbol{\nu}, \Sigma \sim \mathbb{I}_{(-\mathbf{1}, \mathbf{1})} \cdot \mathcal{N}(\boldsymbol{\mu}, \Omega),
$$

where,

$$
\Omega=\left[\sum_{t=2}^{T} d\left(\boldsymbol{\nu}_{t-1}\right) \Sigma^{-1} d\left(\boldsymbol{\nu}_{t-1}\right)\right]^{-1}, \quad \boldsymbol{\mu}=\Omega \cdot \sum_{t=2}^{T} d\left(\boldsymbol{\nu}_{t-1}\right) \Sigma^{-1} \boldsymbol{\nu}_{t} .
$$

In this paper, we omit cumbersome details regarding proposal distributions

240 in $\mathrm{M}-\mathrm{H}$ steps, and we resort to the general purpose JAGS sampler ([35]) for simplicity.

\section{Related work}

Time series of count data are common in studies with diverse medical applications (for instance, see [36, 37] and references therein); hence, Bayesian 245 modelling of such series has been a subject of previous research. Common scalable approaches generally ignore either serial or contemporaneous correlation structures within data; and include standard log-linear models, Gaussian autoregressive approximations and observation-driven state space models. In this work, we will employ univariate Poisson regressions and observation-driven Poisson autoregressive models as a basis for drawing predictive comparisons and discussing congruency in results within Section 5 . For references describing these methods, we refer the reader to 24,33 , or 37.

In summary, Poisson or log-linear regression models allow to model serially uncorrelated count data and will thus permit drawing comparisons with 255 predictive results over large horizons. On the other hand, observation-driven specifications for Poisson autoregressive models offer straightforward and efficient ways to draw inference over short-horizons ([24, 38]). These benefit from easy to calculate likelihoods; however, stationarity and ergodicity properties are hard to derive, and they most importantly suffer from a lack of interpretability 260 on covariates when compared to the parameter-driven alternative discussed in Section 2, 


\section{Data}

The recently deployed electronic task-management system discussed in this paper allows a senior nurse coordinator to triage requests for clinical review and intervention over a team of doctors and clinical support workers; all within the Hospital at Night secondary-care setting in the UK. We note that different organizational models are used for providing $\mathrm{OoH}$ care internationally (39]), and we refer the reader to [40, 41] for information on the setting and research in relation to the source of our data.

In brief, sourced data includes task requests outside the 9:00-17:00 Monday to Friday in hours setting, and incorporates all hours during Bank Holidays. Data belongs to two jointly coordinated hospitals used within the study; therefore, we have complementary sets offering precise information on the $\mathrm{OoH}$ secondary healthcare demand within a geographical region with over 2.5 million

275 residents. Table 1 offers a summary on capacity, total workload and average staff on both sites. In this data set, we observe over 40 different kinds of task requests; most common observations include cannulations, drug prescriptions, clinical reviews and management or blood results interpretations, each accounting for over $10 \%$ of total demand. Also, we notice reasonable amounts of workload in relation to fluids prescriptions, clerking, X-ray requests/reviews or blood test requests.

\begin{tabular}{lccccc}
\hline & \multicolumn{4}{c}{ Average staff in shift (Equivalent in both sites) } \\
\cline { 2 - 5 } & $\begin{array}{c}\text { Senior } \\
\text { doctors }\end{array}$ & $\begin{array}{c}\text { Junior } \\
\text { doctors }\end{array}$ & CSW & $\begin{array}{c}\text { Nurse } \\
\text { coord. }\end{array}$ & \\
\hline \hline 5pm (9am) - 10pm & 1 & 6 & 1 & 1 & \\
10pm - 9am & 1 & 4 & 1 & 1 & \\
\hline & Bed & \multicolumn{4}{c}{ Overall tasks in site } \\
\cline { 2 - 6 } & capacity & 2012 & 2013 & 2014 & 2015 \\
\hline \hline Hospital 1 & 1300 & 57869 & 76461 & 78349 & 78320 \\
Hospital 2 & 1100 & 78353 & 81862 & 96229 & 105142 \\
\hline
\end{tabular}

Table 1: OoH staff levels, capacity and overall workload in both Hospitals; note that day shifts during Weekends begin at 9am.

We find a total of 652,585 entries ranging from January 2012 to December 2015 , and with each of them we obtain attached information including request and completion times, associated ward and medical or surgical speciality, ur285 gency level and assigned staff group. This constitutes a very detailed and complete set of data, rare within management and knowledge engineering studies of this kind. For the study, we isolate requests on the most relevant 14 specialities observed. Table 2 offers an overview of counts over different categories. There, we notice a divergence within median and mean values, along with a high spread 
290 of the week.

\begin{tabular}{lrrrr}
\hline & Min & Median & Max & Mean \& SD \\
\hline \hline Cardiology & 1 & 17 & 58 & $19.29(9.26)$ \\
Clinical haematology & 4 & 18 & 59 & $18.44(6.61)$ \\
Clinical oncology & 5 & 23 & 79 & $25.94(11.86)$ \\
Diabetes & 5 & 22 & 102 & $30.94(20.91)$ \\
General surgery & 15 & 66 & 213 & $80.36(38.37)$ \\
Care for the older people & 10 & 37 & 170 & $54.72(37.07)$ \\
Nephrology and transplants & 4 & 19 & 72 & $21.27(9.76)$ \\
Plastic surgery & 0 & 9 & 36 & $10.14(5.54)$ \\
Respiratory medicine & 14 & 40 & 134 & $50.9(26.21)$ \\
Rheumatology & 0 & 10 & 68 & $14.26(11.34)$ \\
Stroke assessment & 4 & 25 & 85 & $28.97(14.6)$ \\
Trauma and orthopaedic & 1 & 21 & 71 & $23.71(12.09)$ \\
Urology & 0 & 9 & 42 & $10.03(5.71)$ \\
Vascular surgery & 0 & 11 & 57 & $12.44(8.25)$ \\
\hline
\end{tabular}

Table 2: Summary of daily counts in different disciplines, for January 2012 to December 2015. Within parentheses we find standard deviation values.

\section{Results}

Results summarized in this work are obtained from a multivariate sample of 80,000 draws; 20,000 from each of the 4 independent chains run in parallel, all with a 100,000 burn-in phase and showing no significant pairwise correlation within parameters. In this case, $K_{2}=3$ and $K_{3}=5$, and starting values are identical across chains; however, different seeds are used within parallel streams of generated random numbers (for details, see [42]). For predictive purposes, the various models are fitted to data from 2012 to 2014, and results are reported 300 for out-of-sample data.

In the following, we display results that expose the additive value of such electronic tasking information within the local facilities targeted in this study, for the purposes of understanding staff behaviour and managing the delivery of care. Additionally, we summarize conclusions that generalize in time and context, as surveyed among medical consultants across nation-wide healthcare institutions.

\subsection{Periodic patterns, speciality split and bank holiday effect}

In Figure 5 we observe $90 \%$ credible intervals for weekly scaling factors on work-demand, over different medical and surgical specialities; these represent scales on estimated hourly rates of task generation within the studied workplace. 


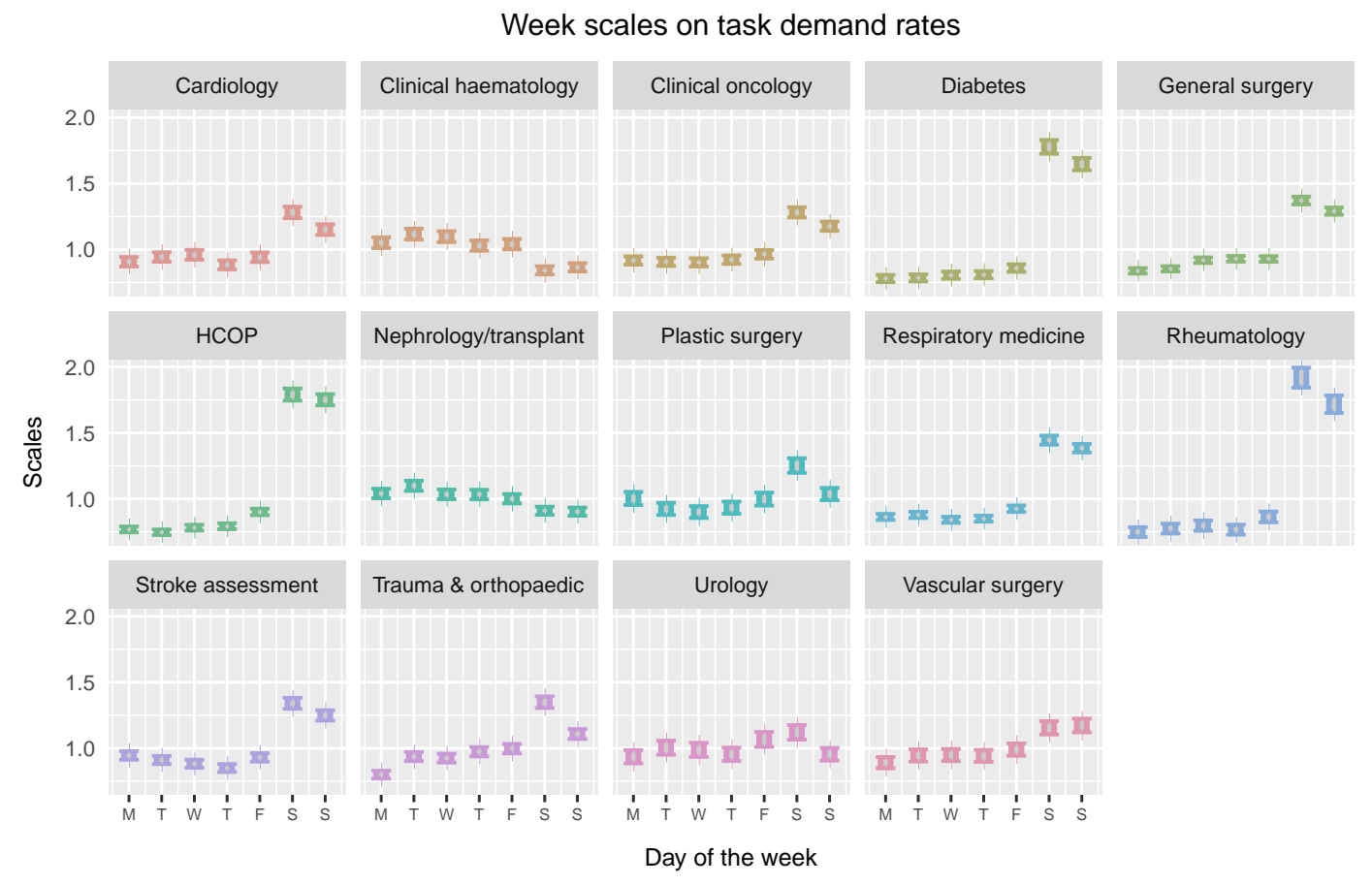

Figure 5: Credible intervals on weekly task-demand scaling factors accross different specialties.

There, we observe varying levels of significant departures from average demand in most specialities, specially over weekends.

An increase in $\mathrm{OoH}$ workload outside business days is mostly triggered by higher proportions of daylight hours; along with sets of leftover duties and 315 routine care or ward round needs usually performed within the in hours setting during working days. Also, the rise in demand is generally more significant on Saturdays; partly, doctors on weekend shifts become familiar with patients and determine needs for treatment alterations during this day; also, Sunday duties may be left for day teams. In general, results sustain claims suggesting that (i) 320 staff behaviour varies according to different shifts/weekdays and (ii) workload is proportionally larger during daylight shifts (see [43] for a related discussion)

However, the variation within business days and weekends is highly dependent on speciality; in this regard, we notice a first split within medical and surgical disciplines. In our study, certain specialities such as haematology or 325 nephrology have external consultants performing ward rounds of their inpatients over weekends, likely explaining some lack of week-round variation in demand. In addition, surgical specialities depend on independent teams dealing with some workload internally, thus not always reporting to centralized management teams. Nevertheless, treatments in surgical specialities are in general 
more elective (cf. 44, 45), and thus allow for better planning; this explains most lack of variation within weekly workload. Extensive research (see [46, 47] and references therein) has raised global concerns regarding a lack of sufficient staff during $\mathrm{OoH}$ shifts over weekends; for instance, mortality rates are known to be proportionally higher. In view of this case study, we notice the ability of electronic tasking-data in order to quantify ward and facility specific needs; also, it is arguable that global weekend staffing should be discipline dependent.

In Figure 6 we observe $90 \%$ credible intervals for bank holiday scaling factors, these are further applied when estimating task-demand during holidays. The similarity between weekend and bank holiday effects is noticeable and not characteristic to this case study (cf. 48]); hence, similar considerations should be made when prioritizing demand and designing rotas.

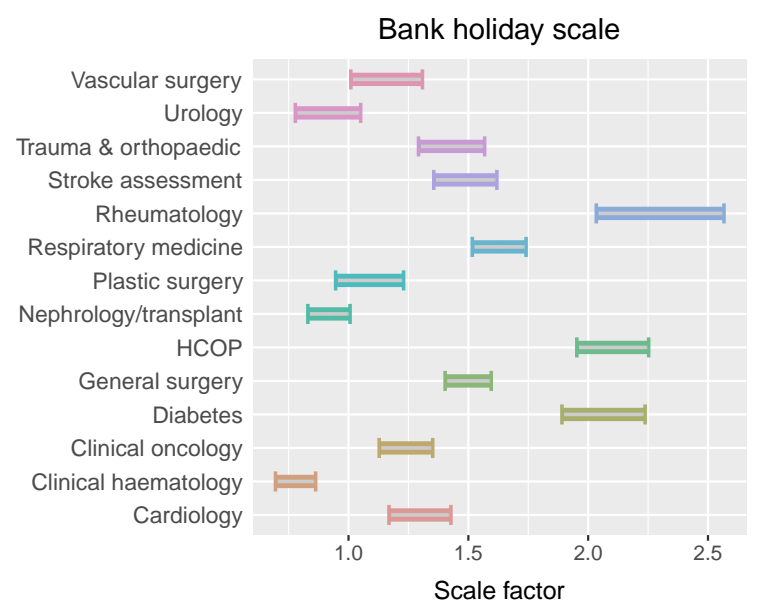

Figure 6: Credible intervals on Bank Holiday task-demand scaling factors accross different specialties

Next, we observe $\% 90$ credible intervals for year-round patterns of OoH taskdemand in Figure 7 There, we again notice diverse levels of vulnerability to seasonality across specialities. The impact is of a lesser relevance to that in weekly scales; with exceptions such as nephrology, susceptible to suffer significant decreases in workload over summer-time, yet uniformly spread over both working and weekend days. There also exist predictable drops in summer workload within specialities where treatment is susceptible to being deferred by patients, such as health care for the older people or diabetes; and significant spikes are observed in categories where workload is vulnerable to admission levels or epidemic trends in certain illnesses. In general, the impact of such seasonal variation is mild (cf. [49) when compared to day-to-day workload; hence, staffing levels must be reasonably balanced all year round, with small exceptions and a clear basis on the split within elective (mostly surgical) and non-elective (mostly medical) disciplines. The elective nature of many surgical operations allows for 


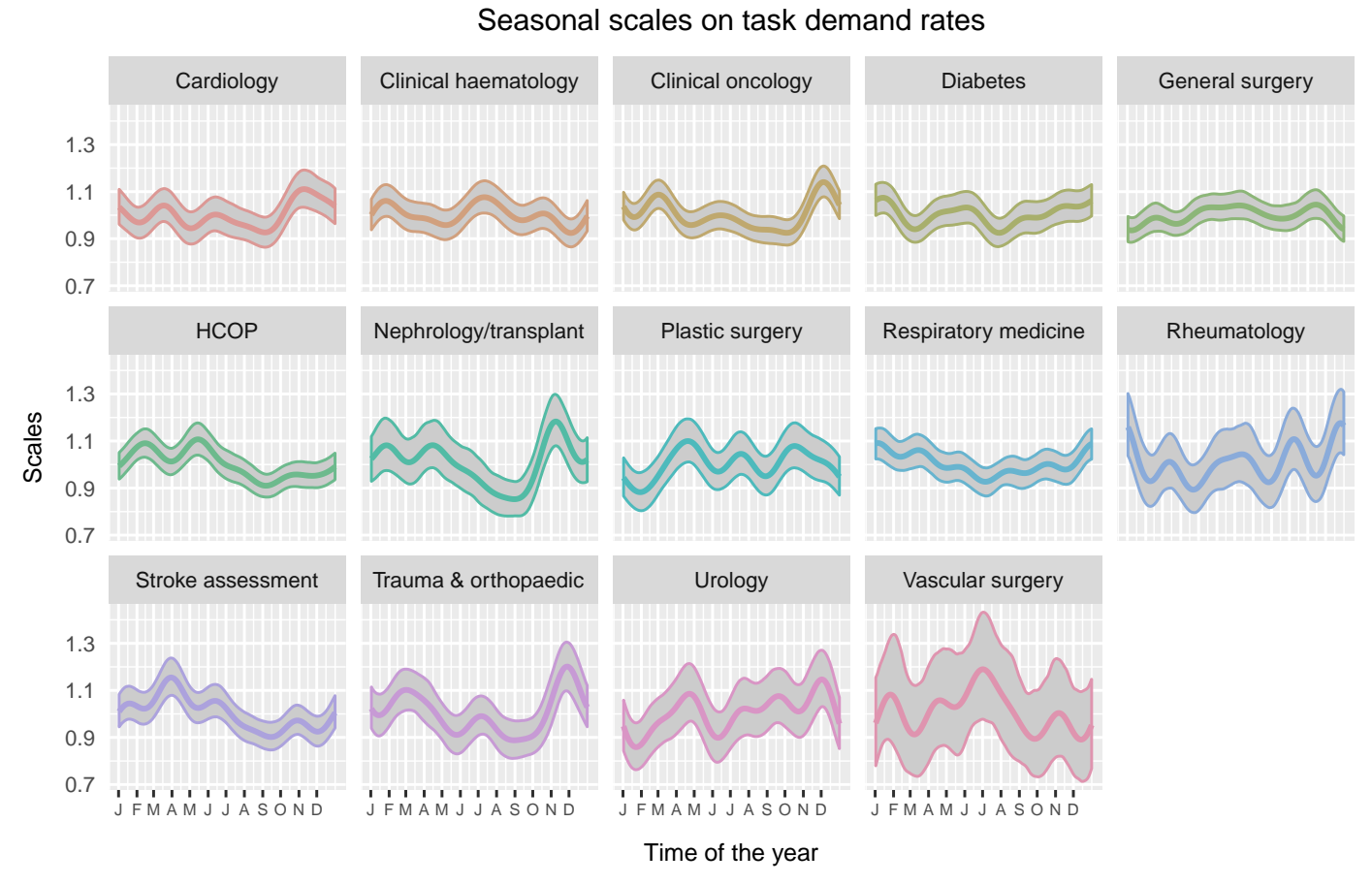

Figure 7: Credible intervals on year-round task-demand scaling factors accross different specialties.

efficient scheduling; hence, it is likely to cause workload alterations aiming to balance year-round stress within the system. Table 3 offers an overview of mean absolute deviations in seasonality scales, around a central tendency of 1 . These statistics allow for direct comparison within disciplines, and manifest the aforementioned variability split within specialities, with the noticeable exception of general surgery.

Finally, we note that seasonal effects on demand are likely to depend on the geographical location of each medical facilitiy; thus, studying localized solutions for demand estimation is of special relevance with aims to design intelligent rota 365 schedules. Thus, we again notice that electronic tasking provides the means for personalizing the study of workload patterns to meet medical facility or ward needs.

\subsection{Serial dependencies and uncorrelated disciplines}

In addition to periodic patterns, we analyse results on temporal and contemporaneous relations within task-demand counts. Figure 8 shows $\% 90$ credible intervals for autoregressive values $\boldsymbol{\delta}$ in (3), which measure latent serial dependencies on marginal trends of task-demand; there, we observe significant positive dependencies across all disciplines. 


\begin{tabular}{lrr}
\hline & Year deviation & Week deviation \\
\hline \hline Cardiology & $0.054(0.011)$ & $0.11(0.009)$ \\
Clinical haematology & $0.044(0.009)$ & $0.09(0.008)$ \\
Clinical oncology & $0.055(0.008)$ & $0.12(0.009)$ \\
Diabetes & $0.046(0.010)$ & $0.34(0.009)$ \\
General surgery & $0.036(0.008)$ & $0.17(0.006)$ \\
Care for the older people & $0.056(0.009)$ & $0.36(0.008)$ \\
Nephrology and transplants & $0.088(0.017)$ & $0.06(0.009)$ \\
Plastic surgery & $0.063(0.013)$ & $0.08(0.011)$ \\
Respiratory medicine & $0.046(0.009)$ & $0.21(0.007)$ \\
Rheumatology & $0.079(0.015)$ & $0.38(0.014)$ \\
Stroke assessment & $0.067(0.011)$ & $0.15(0.009)$ \\
Trauma and orthopaedic & $0.084(0.014)$ & $0.12(0.009)$ \\
Urology & $0.082(0.017)$ & $0.06(0.011)$ \\
Vascular surgery & $0.117(0.025)$ & $0.09(0.012)$ \\
\hline
\end{tabular}

Table 3: Mean absolute deviations in seasonalities. Standard deviations in parentheses are obtained across samples.

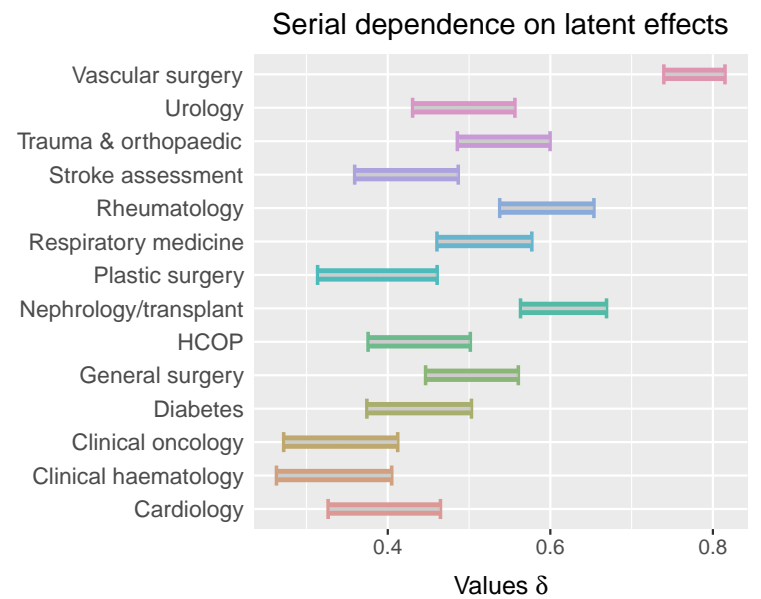

Figure 8: \%90 Credible intervals for autoregressive parameters in vector $\boldsymbol{\delta}$.

Such relation in marginal counts reflects the existence of temporally persistent departures from general trends of expected workload; and emphasizes the importance of short-term planning. Departures may reflect several things; on one hand, the presence of traditional epidemics and associated workload in a medical context, on the other hand, the impact that in hours staffing policy changes exercises on $\mathrm{OoH}$ workload. Note that an increase on team sizes during in hours shifts will likely cause a significant decrease in subsequent workload during $\mathrm{OoH}$; on the contrary, downsizing leads to a higher demand in tasks. 
The inclusion of such autoregressive effects in the model allows to capture these phenomena, hence reducing the bias on inferred seasonality patterns of demand over different categories. Yet, we notice that high serial dependencies observed in Figure 8 may lead to wide intervals of confidence on seasonal patterns; such as in vascular surgery as seen in Figure 7.

Also, Table 4 offers an overview of the latent stationary correlation matrix obtained from (4). We observe a rather homogeneous structure of mostly unrelated pairs across all disciplines, with few exceptions showing very weak 390 contemporaneous dependencies in workload. This suggest it is possible to employ scalable independent forecasts over different disciplines within the studied hospital facilities; yet, we note these relations may not be necessarily meaningful in the general context of $\mathrm{OoH}$ work, but rather respond to work management characteristics within the sites and team analysed in this study. However, general daily counts of task-demand are highly correlated across disciplines; in view of these results, we conclude this is solely due to similarities on seasonal effects across groups of categories.

\begin{tabular}{|c|c|c|c|c|c|c|c|c|c|c|c|c|c|}
\hline & ClHae & ClOnc & Diabe & GeSur & HCOP & Nephr & PlSur & ReMed & Rheum & StAss & Traum & Urolo & VaSur \\
\hline Cardi & $\begin{array}{c}0.059 \\
(0.038)\end{array}$ & $\begin{array}{c}-0.013 \\
(0.038)\end{array}$ & $\begin{array}{c}0.035 \\
(0.038)\end{array}$ & $\begin{array}{c}0.038 \\
(0.036)\end{array}$ & $\begin{array}{c}0.049 \\
(0.037)\end{array}$ & $\begin{array}{c}0.033 \\
(0.038)\end{array}$ & $\begin{array}{c}0.047 \\
(0.041)\end{array}$ & $\begin{array}{l}-0.002 \\
(0.037)\end{array}$ & $\begin{array}{c}0.000 \\
(0.040)\end{array}$ & $\begin{array}{c}0.003 \\
(0.039)\end{array}$ & $\begin{array}{c}0.031 \\
(0.037)\end{array}$ & $\begin{array}{c}0.066 \\
(0.041)\end{array}$ & $\begin{array}{c}0.011 \\
(0.035)\end{array}$ \\
\hline ClHae & & $\begin{array}{c}0.068 \\
(0.039)\end{array}$ & $\begin{array}{l}0.016 \\
(0.037)\end{array}$ & $\begin{array}{c}0.001 \\
(0.034)\end{array}$ & $\begin{array}{c}0.033 \\
(0.036)\end{array}$ & $\begin{array}{c}0.055 \\
(0.037)\end{array}$ & $\begin{array}{c}-0.024 \\
(0.041)\end{array}$ & $\begin{array}{c}0.013 \\
(0.035)\end{array}$ & $\begin{array}{c}0.017 \\
(0.038)\end{array}$ & $\begin{array}{c}0.028 \\
(0.038)\end{array}$ & $\begin{array}{l}-0.006 \\
(0.036)\end{array}$ & $\begin{array}{c}0.008 \\
(0.041)\end{array}$ & $\begin{array}{l}-0.027 \\
(0.032)\end{array}$ \\
\hline ClOnc & & & $\begin{array}{l}-0.009 \\
(0.037)\end{array}$ & $\begin{array}{l}-0.009 \\
(0.034)\end{array}$ & $\begin{array}{l}-0.036 \\
(0.036)\end{array}$ & $\begin{array}{c}0.013 \\
(0.035)\end{array}$ & $\begin{array}{c}0.038 \\
(0.040)\end{array}$ & $\begin{array}{l}-0.020 \\
(0.035)\end{array}$ & $\begin{array}{c}0.007 \\
(0.036)\end{array}$ & $\begin{array}{c}0.010 \\
(0.037)\end{array}$ & $\begin{array}{c}0.001 \\
(0.036)\end{array}$ & $\begin{array}{l}-0.053 \\
(0.040)\end{array}$ & $\begin{array}{l}-0.004 \\
(0.032)\end{array}$ \\
\hline Diabe & & & & $\begin{array}{c}0.065 \\
(0.034)\end{array}$ & $\begin{array}{c}0.066 \\
(0.036)\end{array}$ & $\begin{array}{c}-0.026 \\
(0.037)\end{array}$ & $\begin{array}{c}-0.067 \\
(0.039)\end{array}$ & $\begin{array}{c}\mathbf{0 . 0 8 3} \\
(0.035)\end{array}$ & $\begin{array}{c}\mathbf{0 . 0 8 1} \\
(0.037)\end{array}$ & $\begin{array}{c}0.014 \\
(0.037)\end{array}$ & $\begin{array}{c}0.019 \\
(0.038)\end{array}$ & $\begin{array}{c}-0.001 \\
(0.040)\end{array}$ & $\begin{array}{c}0.068 \\
(0.035)\end{array}$ \\
\hline GeSur & & & & & $\begin{array}{c}\mathbf{0 . 0 8 0} \\
(0.033)\end{array}$ & $\begin{array}{c}-0.029 \\
(0.035)\end{array}$ & $\begin{array}{c}-0.053 \\
(0.036)\end{array}$ & $\begin{array}{c}0.042 \\
(0.034)\end{array}$ & $\begin{array}{c}\mathbf{0 . 0 7 1} \\
(0.035)\end{array}$ & $\begin{array}{c}-0.034 \\
(0.034)\end{array}$ & $\begin{array}{c}\mathbf{0 . 0 8 3} \\
(0.034)\end{array}$ & $\begin{array}{c}0.003 \\
(0.036)\end{array}$ & $\begin{array}{c}\mathbf{0 . 0 9 5} \\
(0.033)\end{array}$ \\
\hline HCOP & & & & & & $\begin{array}{c}0.033 \\
(0.036)\end{array}$ & $\begin{array}{c}-0.014 \\
(0.039)\end{array}$ & $\begin{array}{c}0.009 \\
(0.035)\end{array}$ & $\begin{array}{c}0.058 \\
(0.036)\end{array}$ & $\begin{array}{c}-0.001 \\
(0.035)\end{array}$ & $\begin{array}{c}0.043 \\
(0.035)\end{array}$ & $\begin{array}{c}0.022 \\
(0.037)\end{array}$ & $\begin{array}{c}0.021 \\
(0.033)\end{array}$ \\
\hline Nephr & & & & & & & $\begin{array}{c}0.075 \\
(0.040)\end{array}$ & $\begin{array}{c}-0.048 \\
(0.037)\end{array}$ & $\begin{array}{c}-0.034 \\
(0.038)\end{array}$ & $\begin{array}{c}0.009 \\
(0.038)\end{array}$ & $\begin{array}{c}0.028 \\
(0.037)\end{array}$ & $\begin{array}{c}\mathbf{0 . 0 9 4} \\
(0.039)\end{array}$ & $\begin{array}{l}-0.030 \\
(0.039)\end{array}$ \\
\hline PISur & & & & & & & & $\begin{array}{l}-0.036 \\
(0.037)\end{array}$ & $\begin{array}{l}-0.056 \\
(0.042)\end{array}$ & $\begin{array}{c}0.002 \\
(0.040)\end{array}$ & $\begin{array}{c}0.005 \\
(0.040)\end{array}$ & $\begin{array}{c}\mathbf{0 . 2 0 4} \\
(0.041)\end{array}$ & $\begin{array}{l}-0.055 \\
(0.038)\end{array}$ \\
\hline ReMed & & & & & & & & & $\begin{array}{c}0.020 \\
(0.037)\end{array}$ & $\begin{array}{c}0.016 \\
(0.036)\end{array}$ & $\begin{array}{l}-0.046 \\
(0.035)\end{array}$ & $\begin{array}{c}-0.041 \\
(0.038)\end{array}$ & $\begin{array}{c}0.061 \\
(0.035)\end{array}$ \\
\hline Rheum & & & & & & & & & & $\begin{array}{c}0.023 \\
(0.039)\end{array}$ & $\begin{array}{c}0.032 \\
(0.041)\end{array}$ & $\begin{array}{c}0.003 \\
(0.042)\end{array}$ & $\begin{array}{l}-0.005 \\
(0.038)\end{array}$ \\
\hline StAss & & & & & & & & & & & $\begin{array}{l}-0.001 \\
(0.036)\end{array}$ & $\begin{array}{c}0.017 \\
(0.040)\end{array}$ & $\begin{array}{l}-0.035 \\
(0.033)\end{array}$ \\
\hline Traum & & & & & & & & & & & & $\begin{array}{c}0.011 \\
(0.040)\end{array}$ & $\begin{array}{l}-0.027 \\
(0.037)\end{array}$ \\
\hline Urolo & & & & & & & & & & & & & $\begin{array}{l}-0.060 \\
(0.036)\end{array}$ \\
\hline
\end{tabular}

Table 4: Latent stationary correlation structure obtained from 44. Standard deviations shown within parentheses are computed across samples. Significant correlations at a $5 \%$ confidence level are shown in bold numbers.

\subsection{Predictability of local demand and scheduling horizons}

There exist concerns regarding a growing need for data-driven methods serving as a basis to intelligent rota design; an open debate on the topic can for 
example be found in [50 52 and references therein. In this contribution, we discuss the ability of tasking data and forecasting methods for supporting local and ward level scheduling, prioritisation and management of care delivery.

Under the present framework, we note that it is possible to draw inference on expected work-demand across varied time-horizons. For that matter, either deterministic integration or simulation based forward filtering-procedures may be employed (cf. [26, 28]); thus tracking the evolution of latent dynamics $\boldsymbol{\nu}$ in (3). In both top plots in Figure 9 we observe the evolution of task-demand for disciplines general surgery and rheumatology; there, green bars represent 410 out-of-sample demand during the beginning of year 2015. Additionally, superimposed gray lines represent point estimates for expected demand as inferred from the model in Section 2 predictions over out-of-sample demand correspond to estimates obtained right at the end of 2014. Finally, orange lines represent 1-day ahead predictions obtained from dynamically updating the distribution of the latent process $\boldsymbol{\nu}$, and represent work-demand predictions over the shortest possible horizon.

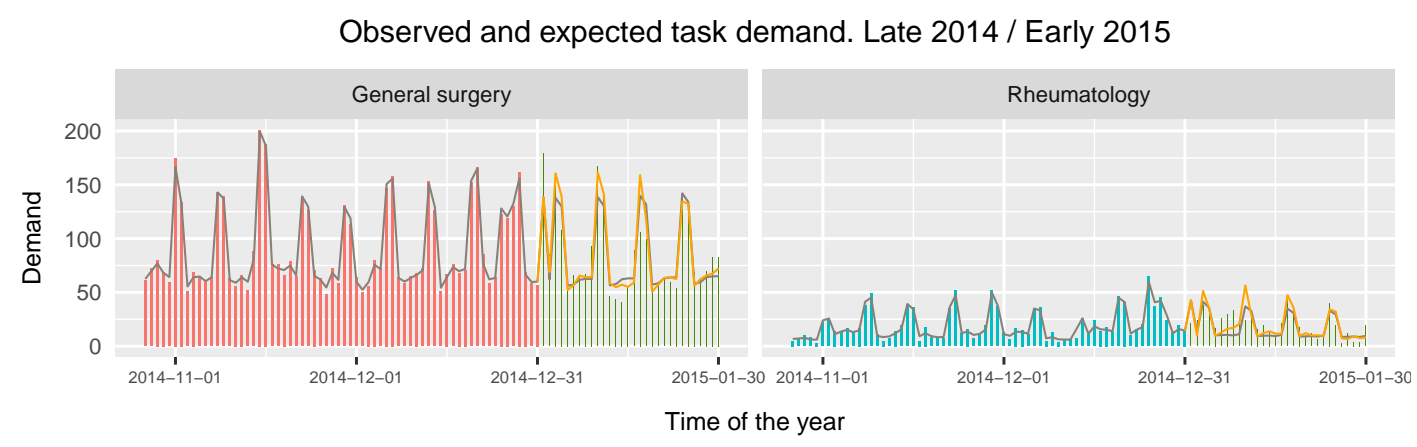

Observed and expected task demand. Late 2014 / Early 2015

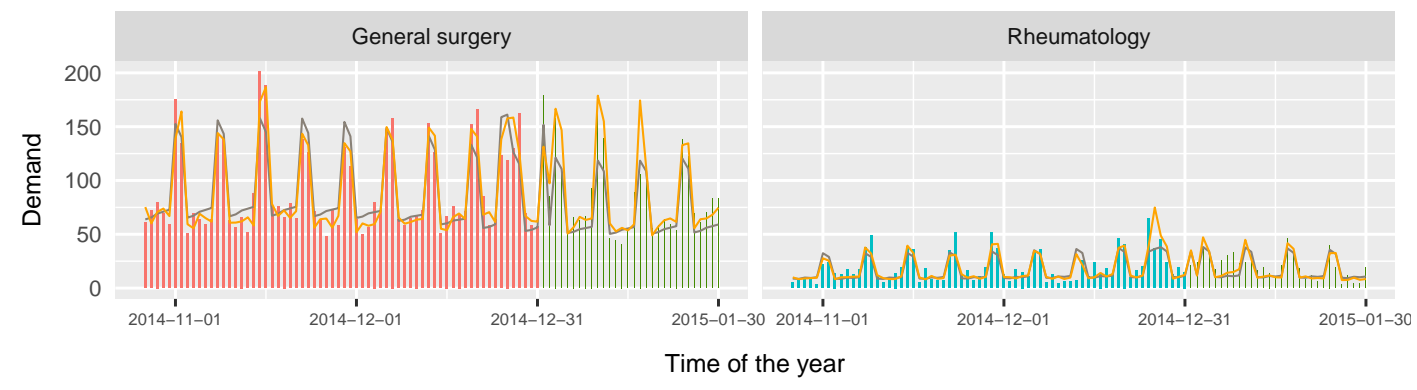

Figure 9: Subset of the evolution of task-demand within disciplines general surgery and rheumatology, green bars represent out-of-sample demand. In top figures, gray lines show estimate predictions drawn at the end of 2014, orange lines are 1-day ahead preditions. In bottom figures, gray lines represent a Poisson regression fit, orange lines 1-day ahead observation-driven AR-Poisson estimates. 
On the other hand, the two bottom plots in Figure 9 present the same evolution of task-demand; yet, superimposed lines offer estimates of task-demand obtained from (i) a Poisson regression model (gray lines) and (ii) an observationdriven Poisson AR(1) model (orange lines, 1-day ahead predictions). Furthermore, Table 5 offers varied measures of predictive accuracy for different fits within the full year 2015 and across all specialities in the study; these include correlation coefficients within estimations and observations, root relative squared errors and relative absolute errors. In order to compute error terms, demand 425 observations and predictions are standardised across medical and surgical categories, thus removing the effect of severe variations in global trends of demand over different specialities.

\begin{tabular}{lccc}
\hline & Cor. coef. & rRSE & RAE \\
\hline \hline 1 day ahead & 0.7409 & $71.98 \%$ & $64.76 \%$ \\
2 days ahead & 0.7113 & $75.98 \%$ & $67.90 \%$ \\
Since end of 2014 & 0.6932 & $78.33 \%$ & $69.97 \%$ \\
Obs-driven AR(1), 1 day ahead & 0.7341 & $72.92 \%$ & $65.52 \%$ \\
Univ. Poisson regression & 0.6837 & $79.53 \%$ & $71.43 \%$ \\
\hline
\end{tabular}

Table 5: Measures of predictive accuracy for various fits in out-of-sample data.

We notice the ability of tasking information in order to produce reasonable estimates on future demand; most importantly, we recall that the granular-

430 ity of such information does not only allow to draw facility-level inference on expected demand, but can also marginalise over sets of specific tasks within precise wards covering particular specialities. In addition, estimates are consistent across models employed and predictive horizons; nevertheless, strong serial dependencies previously observed in Figure 8 force a fast-decreasing predictive 435 power over increasing horizons; again, emphasizing the relevance of short-term scheduling and redesigning of staffing levels. Finally, Figure 10 shows $95 \%$ credible intervals on the evolution of dynamics $\boldsymbol{\nu}$ in (3) across general surgery and rheumatology, and provides additional evidence regarding the loss of information caused by forecasting over large horizons. There, gray intervals represent

440 the MCMC output accounting for data up to the end of year 2014; on the other hand, orange intervals offer 1-day ahead filtered values accounting for data up to the day before each estimate is drawn.

\section{Discussion}

The work in this paper has explored the use of electronic task-management

445 alternatives in combination with modern statistical and machine learning methodology, in order to study the potential for contributions in the design of decision support systems for $\mathrm{OoH}$ workload management in local healthcare facilities. For that matter, the work has examined the general impact of work-demand patterns and contemporaneous dependencies within two major hospitals over a 


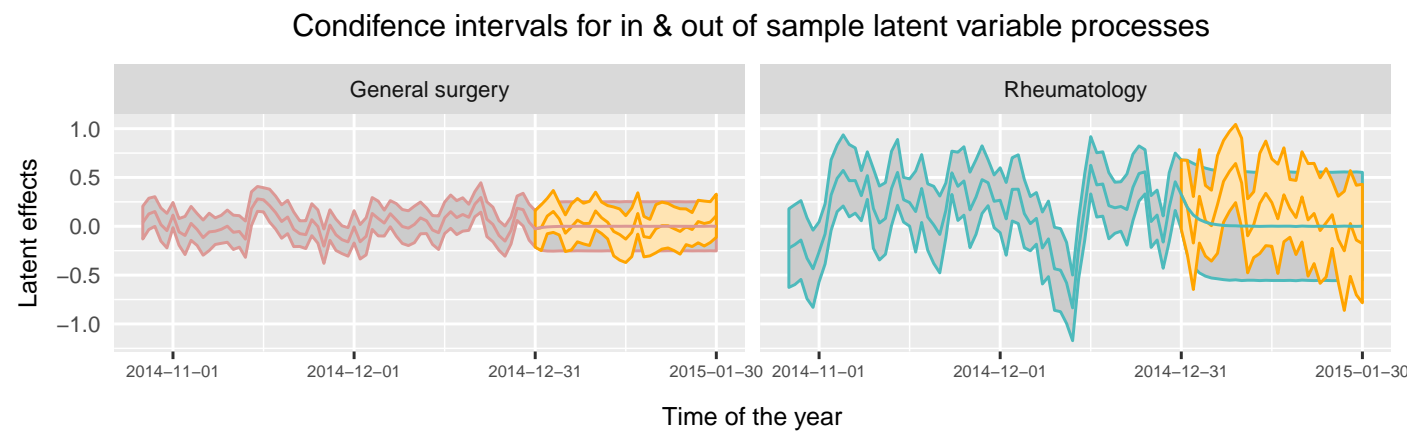

Figure 10: Credible intervals on the evolution of process $\boldsymbol{\nu}$ for general surgery and rheumatology. Orange intervals represent 1-day ahead filtered processes; gray intervals provide MCMC output with data up to 2014.

450 4-year time-window; and has drawn conclusions contributing to open debates discussing needs for intelligent data-driven rotas, speciality-specific staffing variations and the extent of workload disparity within different shifts or working days.

Major contributions in the paper have exhibited the capacity of such tasking data, for providing means to support intelligent data-driven methods for scheduling, prioritisation and management of care delivery. Replacing traditional pager-oriented methods for digital alternatives based on web and mobile interfaces provides means to collect information on workload that has the power of being specific to each ward, speciality, working group or task-type. Also, 460 quantifying demand variability over local facilities may allow for the study of suspected relations with cyclic patterns of staff sickness, norovirus effects on wards, lack of social beds and traditional winter pressures ([53, 54]).

Additionally, scrutinized results in this work have validated several conclusions with practical relevance not restricted to the facilities in the study. In summary,

- Levels of predictable variation in workload and with disparate characteristics within medical and surgical disciplines suggest such split should serve as a basis for weekend rota scheduling.

- Year-round staffing levels should be unaltered. In this regard, imbalances are either mild or statistically not significant, subject to few exceptions.

- Sharp variation within weekday, weekend and bank holiday demand poses a clear argument regarding patients and staff behaving differently according to the day or shift. Also, bank holidays need to be treated as weekends.

- The existence of significant serial relations in task-demand yield fastdecreasing predictive powers over growing horizons; this emphasizes the need for day-to-day organization and consideration of expected workload. 
Finally, a technical limitation in the present modelling approach relates to the latent vector auto-regression structure, which could increase in complexity in order to accommodate a higher order or incorporate serial cross-dependencies within categories, yet increasing the computational requirements in such a highdimensional problem. Also, it would be possible to further relax the restriction on the dispersion of counts using a negative binomial distribution (cf. [28]); or attempt to model contemporaneous correlations employing copulas (31]). Additionally, while we have informed on the impact of seasonality, in order to draw inference on triggering factors (such as admission numbers or care complexity) hospital occupancy information is required.

In conclusion, the work presents a valuable working framework for the study of workload within secondary healthcare institutions during Out of Hours. For this matter, the paper resorts to tasking information from an electronic management system, and results contribute to the quantitative study of care fragmentation, scheduling and team management in healthcare (cf. [17-19]).

\section{Acknowledgements}

We would like to thank the Royal College of Physicians and all the medical and administrative staff; visitors and patients; medical students, junior doctors

and hospital volunteers that took part in the various studies.

This work was supported by The Health Foundation through the Insight 2014 project "Informatics to identify and inform best practice in out of hours secondary care" (7382) and RCUK thought the Horizon Digital Economy Research grants (EP/G065802/1, EP/M000877/1).

[1] C. Roehrig, A. Turner, P. Hughes-Cromwick, G. Miller, When the cost curve bent, pre-recession moderation in health care spending, New England Journal of Medicine 367 (2012) 590-593.

[2] C. Keegan, S. Thomas, C. Normand, C. Portela, Measuring recession severity and its impact on healthcare expenditure, International journal of health care finance and economics 13 (2013) 139-155.

[3] Royal College of Physicians, Hospitals on the edge: the time for action?, RCP London, 2012.

[4] C. Ham, C. Imison, N. Goodwin, A. Dixon, P. South, Where next for the nhs reforms? the case for integrated care, The King's Fund (2011).

[5] J. Cornwell, R. Levenson, L. Sonola, E. Poteliakhoff, E. King's Fund (London, Continuity of care for older hospital patients: a call for action, King's Fund, 2012. 
[18] S. S. Jones, A. Thomas, R. S. Evans, S. J. Welch, P. J. Haug, G. L. Snow, Forecasting daily patient volumes in the emergency department, Academic Emergency Medicine 15 (2008) 159-170. Developing a simulator to help junior doctors deal with night shifts, Contemporary Ergonomics and Human Factors. M. Anderson, Ed. Abingdon: Taylor and Francis (2013) 289-296.

[7] M. Brown, D. Shaw, S. Sharples, I. Le Jeune, J. Blakey, A surveybased cross-sectional study of doctors expectations and experiences of nontechnical skills for out of hours work, BMJ open 5 (2015) e006102.

[8] A. Reeves, M. McKee, S. Basu, D. Stuckler, The political economy of austerity and healthcare: Cross-national analysis of expenditure changes in 27 european nations 1995-2011, Health Policy 115 (2014) 1-8.

[9] R. T. Clarke, A. Pitcher, T. W. Lambert, M. J. Goldacre, UK doctors views on the implementation of the european working time directive as applied to medical practice: a qualitative analysis, BMJ open 4 (2014) e004390.

[10] L. Hallam, Primary medical care outside normal working hours: review of published work, BMJ 308 (1994) 249-253.

[11] C. Salisbury, Evaluation of a general practice out of hours cooperative: a questionnaire survey of general practitioners, BMJ 314 (1997) 1598.

[12] M. B. Christensen, F. Olesen, Out of hours service in denmark: evaluation five years after reform, BMJ 316 (1998) 1502.

[13] C. V. Uden, P. H. Giesen, J. F. Metsemakers, R. P. Grol, Development of out-of-hours primary care by general practitioners (gps) in the netherlands: from small-call rotations to large-scale gp cooperatives., Family Medicine 8 (2006) 565-9.

[14] R. Grol, P. Giesen, C. V. Uden, After-hours care in the united kingdom, denmark, and the netherlands: new models, Health Affairs 25 (2006) 17331737 .

[15] A. Čufar, A. Mrhar, M. Robnik-Šikonja, Assessment of surveys for the management of hospital clinical pharmacy services, Artificial intelligence in medicine 64 (2015) 147-158.

[16] N. B. Peek, Explicit temporal models for decision-theoretic planning of clinical management, Artificial Intelligence in Medicine 15 (1999) 135-154.

[17] A. Rais, A. Viana, Operations research in healthcare: a survey, International transactions in operational research 18 (2011) 1-31.

[6] M. Brown, P. Syrysko, S. Sharples, D. Shaw, I. Le Jeune, E. Fioratou, et al., 
[19] Y. Ben-Shlomo, I. White, P. M. McKeigue, Prediction of general practice workload from census based social deprivation scores., Journal of epidemiology and community health 46 (1992) 532-536.

[20] S. Sharples, M. Brown, J. Pinchin, J. Blum, A. Nagiyev, B. Ryan, et al., Ubiquitous technologies for capture of real-world performance, in: Proceedings 19th Triennial Congress of the IEA, Editors G. Lindgaard \& D. Moore, International Ergonomics Association, volume 9, 2015, p. 14.

[21] C. Larkin, R. Valand, P. Syrysko, R. Harris, D. Shaw, M. Brown, et al., 'night shift': A task simulation to improve on-call prioritisation, selfmanagement, communication, and route planning skills, in: Interactive Technologies and Games (iTAG), 2014 International Conference on, IEEE, 2014, pp. 59-62.

[22] I. Perez, J. Pinchin, M. Brown, J. Blum, S. Sharples, Unsupervised labelling of sequential data for location identification in indoor environments, Expert Systems with Applications 61 (2016) 386 - 393.

[23] R. A. Davis, W. Dunsmuir, Y. Wang, Modeling time series of count data, Statistics Textbooks \& Monographs 158 (1999) 63-114.

[24] R. C. Jung, M. Kukuk, R. Liesenfeld, Time series of count data: modeling, estimation and diagnostics, Computational Statistics \& Data Analysis 51 (2006) 2350-2364.

[25] D. Koller, N. Friedman, Probabilistic graphical models: principles and techniques, MIT press, 2009.

[26] N. Chapados, Effective bayesian modeling of groups of related count time series, Proceedings of the 31st International Conference on Machine Learning, Ed. N. Lawrence, Journal of Machine Learning Research: W\&CP, Microtome Publishing (2014).

[27] A. Acharya, J. Ghosh, M. Zhou, Nonparametric bayesian factor analysis for dynamic count matrices, Proceedings of the 18th International Conference on Artificial Intelligence and Statistics, Ed. N. Lawrence, Journal of Machine Learning Research: W\&CP, Microtome Publishing (2015).

[28] R. C. Jung, R. Liesenfeld, J.-F. Richard, Dynamic factor models for multivariate count data: An application to stock-market trading activity, Journal of Business \& Economic Statistics 29 (2011) 73-85.

[29] P. T. Brandt, T. Sandler, A bayesian poisson vector autoregression model, Political Analysis 20 (2012) 292-315.

[30] S. L. Zeger, A regression model for time series of counts, Biometrika 75 (1988) 621-629. 
[31] A. Heinen, E. Rengifo, Multivariate autoregressive modeling of time series count data using copulas, Journal of Empirical Finance 14 (2007) 564-583.

[32] B. Jørgensen, S. Lundbye-Christensen, P.-K. Song, L. Sun, A state space model for multivariate longitudinal count data, Biometrika 86 (1999) 169181.

[33] L. Held, M. Höhle, M. Hofmann, A statistical framework for the analysis of multivariate infectious disease surveillance counts, Statistical modelling 5 (2005) 187-199.

[34] S. Chib, R. Winkelmann, Markov chain monte carlo analysis of correlated count data, Journal of Business \& Economic Statistics 19 (2001) 428-435.

[35] M. Plummer, Jags: A program for analysis of bayesian graphical models using gibbs sampling, in: Proceedings of the 3rd International Workshop on Distributed Statistical Computing, Editors K. Hornik, F. Leisch \& A. Zeileis, Technische Universitt Wien, Vienna, volume 124, 2003, p. 125.

[36] K. Bhaskaran, A. Gasparrini, S. Hajat, L. Smeeth, B. Armstrong, Time series regression studies in environmental epidemiology, International journal of epidemiology (2013) dyt092.

[37] J. Schwartz, C. Spix, G. Touloumi, L. Bachárová, T. Barumamdzadeh, et al., Methodological issues in studies of air pollution and daily counts of deaths or hospital admissions., Journal of epidemiology and community health 50 (1996) S3-11.

[38] J. Durbin, S. J. Koopman, Time series analysis of non-gaussian observations based on state space models from both classical and bayesian perspectives, Journal of the Royal Statistical Society: Series B (Statistical Methodology) 62 (2000) 3-56.

[39] L. Huibers, P. Giesen, M. Wensing, R. Grol, Out-of-hours care in western countries: assessment of different organizational models, BMC health services research 9 (2009) 1.

[40] D. Beckett, C. Gordon, R. Paterson, S. Chalkley, C. Stewart, M. C. Jones, M. Young, D. Bell, Improvement in out-of-hours outcomes following the implementation of hospital at night, QJM 102 (2009) 539-546.

[41] J. D. Blakey, D. Guy, C. Simpson, A. Fearn, S. Cannaby, P. Wilson, D. Shaw, Multimodal observational assessment of quality and productivity benefits from the implementation of wireless technology for out of hours working, BMJ open 2 (2012) e000701.

[42] P. L'ecuyer, R. Simard, E. J. Chen, W. D. Kelton, An object-oriented random-number package with many long streams and substreams, Operations research 50 (2002) 1073-1075. 
[52] C. Kovner, P. J. Gergen, Nurse staffing levels and adverse events following surgery in u.s. hospitals, Image: the Journal of Nursing Scholarship 30 (1998) 315-321.

655

[43] H. Fukuda, M. Takahashi, H. Airto, Nurses workload associated with 16-h night shifts on the 2-shift system. i: Comparison with the 3-shift system, Psychiatry and Clinical Neurosciences 53 (1999).

[44] W. B. Robb, M. J. O'Sullivan, A. E. Brannigan, D. J. Bouchier-Hayes, Are elective surgical operations cancelled due to increasing medical admissions?, Irish Journal of Medical Science 173 (2004) 129-132.

[45] F. Dexter, L. ONeill, Data envelopment analysis to determine by how much hospitals can increase elective inpatient surgical workload for each specialty, Anesthesia \& Analgesia 99 (2004) 1492-1500.

[46] R. Barba, J. Losa, M. Velasco, C. Guijarro, G. G. de Casasola, A. Zapatero, Mortality among adult patients admitted to the hospital on weekends, European Journal of Internal Medicine 17 (2006) 322 - 324.

[47] L. Schmulewitz, A. Proudfoot, D. Bell, The impact of weekends on outcome for emergency patients, Clinical Medicine 5 (2005) 621-625.

[48] R. S. Kovats, S. Hajat, P. Wilkinson, Contrasting patterns of mortality and hospital admissions during hot weather and heat waves in greater london, uk, Occupational and environmental medicine 61 (2004) 893-898.

[49] A. J. Elliot, K. W. Cross, D. M. Fleming, Acute respiratory infections and winter pressures on hospital admissions in england and wales 1990-2005, Journal of public health 30 (2008) 91-98.

[50] L. H. Aiken, S. P. Clarke, D. M. Sloane, Hospital staffing, organization, and quality of care: Cross-national findings, Nursing Outlook 50 (2002) $187-194$.

[51] C. Kovner, C. Jones, C. Zhan, P. J. Gergen, J. Basu, Nurse staffing and postsurgical adverse events: An analysis of administrative data from a sample of u.s. hospitals, 19901996, Health Services Research 37 (2002) 611-629.

[53] K. Fullerton, V. Crawford, The winter bed crisisquantifying seasonal effects on hospital bed usage, Qjm 92 (1999) 199-206.

[54] C. Vasilakis, E. El-Darzi, A simulation study of the winter bed crisis, Health Care Management Science 4 (2001) 31-36. 